\author{
Júlia Borràs \\ Department of Mechanical Engineering \\ and Materials Science, \\ Yale University, \\ New Haven, CT 06520 \\ e-mail: julia.borrassol@yale.edu \\ Aaron M. Dollar \\ Department of Mechanical Engineering \\ and Materials Science, \\ Yale University, \\ New Haven, CT 06520 \\ e-mail: aaron.dollar@yale.edu
}

\section{Actuation Torque Reduction in Parallel Robots Using Joint Compliance}

\begin{abstract}
This work studies in detail how the judicial application of compliance in parallel manipulators can produce manipulators that require significantly lower actuator effort within a range of desired operating conditions. We propose a framework that uses the Jacobian matrices of redundant parallel manipulators to consider the influence of compliance both in parallel with the actuated joints as well as the passive joints, greatly simplifying previous approaches. We also propose a simple optimization procedure to maximize the motor force reduction for desired regions of the workspace and range of external forces. We then apply the method to a Stewart-Gough platform and to a 3-URS (universal rotational and spherical joint) manipulator. Our results show that parallel manipulators with tasks that involve a preferred external force direction, as for instance, big weights in the platform, can see large reductions in actuator effort through the judicial use of compliant joints without significantly losing rigidity. [DOI: 10.1115/1.4026628]
\end{abstract}

Keywords: compliant joints, redundant parallel manipulators, static analysis

\section{Introduction}

The use of compliant joints in robotics has become popular in the last few decades for several different reasons. Springs in series with actuators has been widely studied and implemented, most notably through series elastic actuators [1]. This arrangement can allow for the more reliable implementation of force control, increase the adaptability of the mechanism to external contacts, and in the case of transmissions with low back-drivability, can allow a contact force to be applied at the output without power applied to the actuator. Compliance in parallel systems can be used to allow the underconstrained degrees of freedom in an underactuated mechanism to reconfigure in presence of an external contact $[2,3]$.

We focus our attention on compliant joints used in parallel manipulators. Recently, several authors have studied how to model the stiffness matrix of parallel manipulators that use passive compliant joints and/or springs in series with the actuators [4-7]. In this kind of compliant manipulators, the forward kinematics have to be solved simultaneously with the static analysis [8], as each configuration will depend on the external applied force.

In this paper, we study the effect of passive compliant joints (such as flexure-based or spring-loaded joints) and springs in parallel with the actuators to reduce actuation force. Prior work has studied how to design parallel manipulators adding certain compliant elements that lead to a constant potential energy at any configuration, when no external force is applied (only gravity) [9-11]. In such gravity compensated manipulators, the actuation force is greatly reduced, resulting in significant improvement of the control and energy efficiency. However, there is not a general methodology to design such manipulators. Their challenging design process consists in expressing the potential energy as a function of a minimal set of coordinates. The condition for static balance is then obtained by imposing some coefficients depending on design parameters to be zero [9], but a feasible solution is not always possible and requires a complex ad hoc initial design. For instance, Ref. [9] shows a statically balanced six degrees of

Contributed by the Mechanisms and Robotics Committee of ASME for publication in the Journal of MeChanisms and RoBotics. Manuscript received April 22, 2013; final manuscript received December 20, 2013; published online March 4, 2014. Assoc. Editor: Andreas Müller. freedom manipulator using legs formed by compliant parallelogram mechanisms.

In this paper, we show how the springs in parallel with the actuated and passive joints can significantly reduce the force exerted by the motors in the presence of certain external forces without requiring such complex design process. Some of the big advantages of parallel manipulators over their serial counterparts are their high stiffness, accuracy and, their ability to support much higher loads. One of the major drawbacks is their small workspace, which are even more reduced when considering singularities [12] and the limits of the forces the motors can exert $[13,14]$. In this context, parallel platforms can greatly benefit from the use of springs in parallel with the actuators to help to reduce the required motor forces, enhancing the size of the usable workspace and reducing the size of the actuators.

Note that such manipulators will not be compliant. Indeed, the rigidity of the manipulator that uses compliance in the passive joints or compliance in parallel with the motors is not greatly modified, because the stiffness of the motors is usually several orders of magnitude bigger than the stiffness of the springs. Therefore, such passive compliance was usually ignored for the compliant analysis. However, in Ref. [15] they shown how taking the passive compliance into account can increase the accuracy of the model.

Our work is not focused on stiffness analysis of manipulators [4-7,16-19], but on the analysis of how compliance in parallel with the joints modifies the load on the motors. Other works have shown that springs can reduce the energy consumption by minimizing the sum of the actuation torques through motion trajectories for serial manipulators [20] or walking [21] and running robots [22]. We show that springs are also useful for reducing actuation torque for parallel mechanisms.

Our framework takes into account compliance in the passive joints and compliance in the actuated ones in parallel with the motors. We consider any joint with compliance as an active joint, for the purpose of computing the Jacobian matrix. In other words, our approach considers the parallel manipulator as redundant and uses screw theory to obtain the Jacobian matrix of the redundant manipulator that defines the transmission relationship between torques and external wrenches on the platform [23]. Using an appropriate definition of the torque for each type of joint, we can quantify the reduction or increase of torque that the motors have 


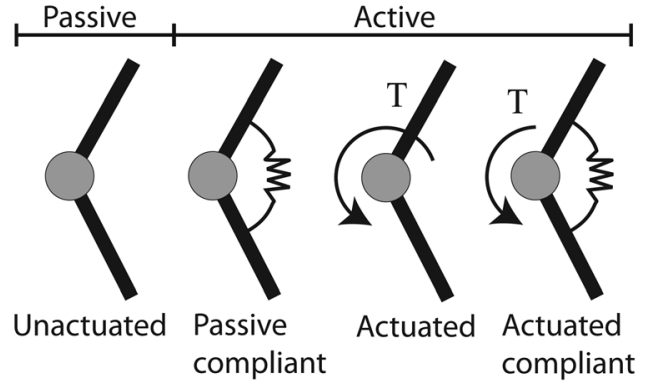

Fig. 1 Classification of joints with and without compliance. In the parallel robot literature, any nonactuated joint is called passive, but as the passive compliant joint exerts a torque, we have to consider it as active for the static analysis.

to exert to overcome a given external applied wrench thanks to the springs located both in actuated and passive joints.

With a different definition of the compliant actuated joints, our framework is equivalent to the one proposed in Ref. [6]. However, the use of a single redundant Jacobian matrix greatly simplifies the static equilibrium equations.

Our preliminary work on this topic [24] showed that significant torque reduction can be obtained with springs only in the passive joints for particular poses. Here, we generalize the results to take into account all the workspace and we propose a simple optimization procedure to minimize the total torque exerted by all the motors through all the workspace for a range of forces. As a result, we will see how the manipulator can reduce the required torque up to $84 \%$ for an important percentage of the workspace under the effect of certain range of forces, and also significantly increase of the usable workspace due to motor force limits.

We begin this paper by defining the type of compliant joints we will consider, and reviewing of the mathematical framework to study the static analysis of redundant parallel manipulators. In Sec. 3, we define the relationship between motor force and the compliant force, followed by the introduction of the proposed optimization method to design and measure the influence of the springs. Sections 4 and 5 apply the proposed method to two example manipulators, a Stewart-Gough platform and a 3-URS manipulator, and Sec. 6 discusses the obtained results and summarizes the conclusions and directions for future work.

\section{Parallel Platforms With Compliant Joints}

2.1 The Joints of a Compliant Parallel Manipulator. By construction, any configuration of the joints in a parallel manipulator satisfies the constraint that the distances between the attachments of each leg at the platform are constant; i.e., kinematic constraints. To ensure they are satisfied, most manipulators leave some of the joints free to move (passive joints), so that the joints controlled by the motors (active joints) determine the pose of the platform, while the passive joint angles automatically adapt their value to hold the kinematic constraints.

A passive joint does not produce force/torque reactions and thus, it does not appear in the static equations. On the contrary, a compliant passive joint exerts force and thus, is typically treated as an active joint [4]. We distinguish between 4 types of joints, depicted in Fig. 1. Passive joints are unactuated (free to move). Passive compliant joints are also unactuated, but have a spring that exerts torques in accordance to their configuration and spring stiffness constants. Actuated joints are controlled by a motor and, in static equilibrium, they exert torques reacting to an external force applied at the platform that is transmitted through the legs to the joints. Finally, compliant actuated joints have the motor and the spring in parallel, such that the resultant torque is the sum of torque resulting from the motor and the torque resulting from the spring.
All the joints exerting torque are called active. Depending on the type of joint, the total force/torque they exert is

$$
\begin{aligned}
& \tilde{\tau}_{i}=\tau_{i}-k_{i}\left(\theta_{i}-\delta_{i}\right) \text { for actuated complaint, } \\
& \tilde{\tau}_{i}=-k_{i}\left(\theta_{i}-\delta_{i}\right) \text { for passive complaint, and } \\
& \tilde{\tau}_{i}=\tau_{i} \text { for actuated joints, }
\end{aligned}
$$

where $\tau_{i}$ is the torque/force exerted by the motor and ki is the stiffness contant of the spring. Previous approaches have computed the influence of the passive compliant joints by adding an extra term in the static equations that depend on the Jacobian matrix relating the passive and the active joints [6]. Here, we propose to consider compliant parallel manipulators as redundant manipulators to compute its Jacobian matrix. In Sec. 2.2, we review the method of deriving the Jacobian matrix of parallel manipulators using screw theory.

2.2 The Jacobian Matrix of Redundant Parallel Manipulators. Consider a parallel manipulator in $\mathbb{R}^{3}$. Its platform can be moved in a maximum of 6 degrees of freedom (DOF), 3 for position, and 3 for orientations, defined in a vector $x \in \mathbb{R}^{6}$. Depending on the number of legs, links, and joints, we can compute the mobility of the manipulator, $n$, using the Grübler-Kutzbach criterion. If $n<6$, the manipulator is called lower mobility [25], that is, the workspace of the manipulator consists of a $n$ dimensional subspace of the six-dimensional task space. If the mobility is higher than 6 , the manipulator workspace is still six-dimensional, but it has kinematic redundancy [26].

For simplicity, we consider only 1 DOF joints. In other words, a universal (spherical) joint is considered as two (three) rotational joints with intersecting axes. We assume that the manipulator has full mobility $(n=6)$. Then, if the manipulator has $l$ equal legs, each leg has to have 6 joints to allow the full mobility of the platform. Therefore, the total number of joints is $m=6 l$. Let

$$
\boldsymbol{\Theta}=\left(\theta_{11}, . ., \theta_{6 l}\right)^{T}
$$

be the vector of all the joint angles. Only $n$ of them are independent and determine the position of the platform. Let $n_{a}$ be the number of joints actuated by a motor. If $n_{a}=n$, the manipulator is called fully actuated. If $n_{a}>n$, the manipulator is said to be redundantly actuated. Note that the number of actuated joints must be at least the same as the mobility, otherwise, the manipulator would have uncontrolled free DOFs.

The twist acting on the platform $\boldsymbol{T}=(\boldsymbol{v}, \boldsymbol{\Omega})$, composed of linear velocity and angular velocity, can be written as linear combination of the twists defined by each of the joints of the legs $[27,28]$. Mathematically, that is

$$
\boldsymbol{T}=\sum_{j=1}^{6} \dot{\theta}_{i j} \$_{i j}, \quad i=1, \ldots, l
$$

where $\$_{i j}$ corresponds to the screw associated to the $j$ th joint of the $i$ th leg and $\dot{\theta}_{i j}$ is the velocity of the $j$ th joint on the $i$ th leg. For a rotational joint located at $\boldsymbol{p}$ with an axis of rotation along $z$, its associated screw is $(\boldsymbol{z}, \boldsymbol{p} \times \boldsymbol{z})$. For a prismatic joint along $z$, its associated screw is $(\mathbf{0}, \mathbf{z})$.

In each leg, let $g$ of the joints be active (that is, either compliant or actuated), while the rest $(6-g)$ are free to move. To eliminate the passive variables from Eq. (3), we compute the system of screws that are reciprocal to the passive joint screws. Two screws $\$_{1}=\left(\boldsymbol{v}_{1}, \boldsymbol{w}_{1}\right)$ and $\$_{2}=\left(\boldsymbol{v}_{2}, \boldsymbol{w}_{2}\right)$ are reciprocal when its reciprocal product is zero, that is,

$$
\$_{1} * \$_{2}=\boldsymbol{v}_{1} \cdot \boldsymbol{w}_{2}+\boldsymbol{w}_{1} \cdot \boldsymbol{v}_{2}=0
$$

There are $6-(6-g)=g$ screws reciprocal to the passive joints $[29,30]$. Let $\$_{\text {rik }}$, for $k=1, \ldots, g$, be the system of screws reciprocal to the passive joint screws. If we apply the reciprocal product 
at both sides of the equations in Eq. (3), we can rewrite the system as

$$
\boldsymbol{J}_{T_{i}} \boldsymbol{T}=\boldsymbol{J}_{\Theta i}\left(\begin{array}{c}
\dot{\theta}_{i 1} \\
\vdots \\
\dot{\theta}_{i h}
\end{array}\right)
$$

where

$$
\boldsymbol{J}_{T_{i}}=\left(\begin{array}{c}
\$_{r i 1}^{T} \\
\vdots \\
\$_{r i g}^{T}
\end{array}\right)
$$

and

$$
\boldsymbol{J}_{\Theta i}=\left(\begin{array}{ccc}
\$_{r i 1} * \$_{i 1} & \ldots & \$_{r i 1} * \$_{i g} \\
\vdots & \ddots & \vdots \\
\$_{r i g} * \$_{i 1} & \ldots & \$_{r i g} * \$_{i g}
\end{array}\right)
$$

Writing the $l$ equations in a single matrix system form, we get

$$
\boldsymbol{J}_{T} \boldsymbol{T}=\boldsymbol{J}_{\Theta}\left(\begin{array}{c}
\dot{\theta}_{i 1} \\
\vdots \\
\dot{\theta}_{i h}
\end{array}\right)
$$

where

$$
\boldsymbol{J}_{T}=\left(\begin{array}{c}
\boldsymbol{J}_{T 1} \\
\vdots \\
\boldsymbol{J}_{T l}
\end{array}\right)
$$

is a $\lg \times 6$ matrix and

$$
\boldsymbol{J}_{\Theta}=\left(\begin{array}{ccc}
J_{\Theta 1} & \ldots & 0 \\
\vdots & \ddots & \vdots \\
0 & \ldots & J_{\Theta l}
\end{array}\right)
$$

is $l g \times \lg$. Then, the Jacobian matrix of the parallel manipulator is usually defined as $\boldsymbol{J}=\boldsymbol{J}_{\Theta}^{-1} \boldsymbol{J}_{T}$, and the relationship between the platform twist and the joint velocities as

$$
J T=\dot{\Theta}
$$

A wrench acting the platform is composed by a force and a moment $\boldsymbol{W}=(\boldsymbol{f}, \boldsymbol{m})$. The above equation is used with the principle of virtual work to obtain the static equilibrium equations [27]. As a result, the relationship between the torques exerted by the joints and the transmitted wrench on the platform, is

$$
\boldsymbol{W}=\boldsymbol{J}^{T} \tau
$$

where $\boldsymbol{J}^{T}$ is the transposed of the matrix in Eq. (11), and it has dimensions $6 \times l g$. The system is then in static equilibrium when the external applied wrenches $\boldsymbol{W}$ satisfies

$$
\boldsymbol{W}=-\boldsymbol{J}^{T} \tau
$$

\section{Measuring the Influence of Compliance}

3.1 Motor Force and Compliance. This work studies in detail how springs in parallel with the motors can affect the performance of the manipulator and the required motor output.

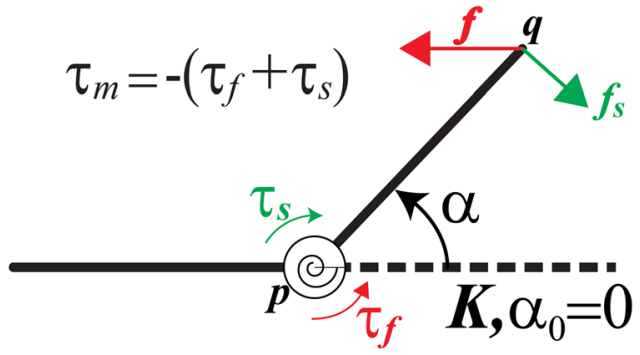

Fig. 2 Simple example of the combination of actuation torque exerted by the motor $\tau_{m}$, compliant torque exerted by the spring $\tau_{s}$, and reaction torque to the external force $\tau_{f}$

In Fig. 2, we show two links connected by an actuated revolute joint with a torsional spring in parallel. If no external force is applied, the motor will need to overcome only the torque exerted by the spring. In other words, in a static equilibrium configuration, if $\tau_{s}=-K\left(\alpha-\alpha_{0}\right)$ is the spring torque, where $K$ is the stiffness constant and $\alpha_{0}$ is resting configuration, the motor exerts a torque $\tau_{m}=-\tau_{s}$ to maintain the link at angle $\alpha$. An external force $f$ applied on the tip transmits a torque on the joint of magnitude $\tau_{f}=|(\boldsymbol{q}-\boldsymbol{p}) \times \boldsymbol{f}|$, such that the torque the motor needs to exert to achieve static equilibrium is $\tau_{m}=-\left(\tau_{s}+\tau_{f}\right)$.

In this very simple case, it is obvious that the motor will reduce the exerted torque when the signs of the motor and spring torques are opposite, and there is always a configuration for which it can be reduced to 0 .

For a parallel manipulator with several links and joints, we represent all the torques exerted by the springs in a vector $\tau_{c}=\left(-K_{i}\left(\theta_{i}-\delta_{i}\right)\right)^{T}$, where $K_{i}$ is the spring stiffness constant of the spring in the joint $i$ and $\delta_{i}$ its resting configuration (linear or torsional depending on the type of joint). Similarly, we write the forces/torques exerted by the motors in a vector $\tau_{m}=\left(\tau_{i}\right)^{T}$. The vector of forces/torques transmitted by an external wrench $\boldsymbol{W}=(\boldsymbol{f}, \boldsymbol{m})^{T}$ is given by the relationship in Eq. (12), $\boldsymbol{W}=\boldsymbol{J}^{T} \tau_{F}$. The relationship between the vector of all the joint forces/torques is, analogously as the previous case, $\tau_{m}=-\left(\tau_{s}+\tau_{F}\right)$. To avoid inverting the Jacobian matrix, we can multiply at both sides of the equality by $\boldsymbol{J}^{T}$, leading to

$$
-\boldsymbol{W}=\boldsymbol{J}^{T} \tau_{m}+\boldsymbol{J}^{T} \tau_{\boldsymbol{s}}
$$

This expression is equivalent to substituting the values of the torques given in Eq. (1) into the expression in Eq. (13). Note that, if the joint $j$ is actuated without compliance, the corresponding $j$ component in the vector $\tau_{s}$ is zero. Similarly, if the joint $j$ is passive compliant, the corresponding position $j$ in the vector $\tau_{m}$ is zero. We believe that this equation is simpler than the model introduced in Ref. [6], and they are equivalent.

Also, for a fully-actuated manipulator, the number of motors is 6 and thus, there are only 6 nonzero elements in the vector $\tau_{m}$. Let $\tau_{m}^{\prime}$ be the motor torques vector without the zero components. Then, we can rewrite the system as

$$
-\boldsymbol{F}=\boldsymbol{J}_{m} \boldsymbol{\tau}_{\boldsymbol{m}}^{\prime}+\boldsymbol{J}^{T} \tau_{\boldsymbol{s}}
$$

where $\boldsymbol{J}_{m}$ is a $6 \times 6$ matrix obtained from $\boldsymbol{J}^{T}$ eliminating the columns corresponding to 0 torque. This system states a one-to-one correspondence between external applied wrench and motor forces/torques.

Given an external wrench, in each configuration we can solve the system for $\tau_{m}$. Let us call a solution of the system $\tau_{C}(C$ stands for compliance). Then, $\tau_{C}$ gives us the torques exerted by the motors for a mechanism including springs. We call $\tau_{\mathrm{NC}}$ the solution of the same system with $\tau_{s}$ set to zero. Then, $\tau_{\mathrm{NC}}$ are the torques done by the motor for a mechanism without compliant joints. 
Thus, comparing the values $\tau_{\mathrm{NC}}$ and $\tau_{C}$ we can quantify the decrease or increase of the torque done by the motors in a given configuration and for a given force.

Singular poses are defined by those configurations where $\operatorname{det}\left(\boldsymbol{J}_{m}\right)=0$. Near such configurations, the values of the actuation torques can be very high even with small external applied forces. Therefore, in practice, the borders of the static workspace (or reachable workspace) are defined by those configurations that reach the limit on the torques that the motors can exert [13]. Thus, with Eq. (15) we have shown that adding springs in parallel with the joints can change those limits.

We will show how to design the spring parameters to maximize the number of configurations where the springs help to reduce the torque exerted by the motors, increasing then the efficiency of the manipulator. We will also show how can we quantify and visualize the configurations where the torque is reduced and for which range of forces.

3.2 Measuring Compliance Influence. In a configuration $P$, we define the overall force/torque exerted by the motors as the sum of the squares of all the force/torques exerted by the motors for both the mechanism with springs or without

$$
\begin{aligned}
& \mathrm{OT}_{\mathrm{CP}}=\tau_{C}^{T} \tau_{C} \\
& \mathrm{OT}_{\mathrm{NCP}}=\tau_{\mathrm{NC}}^{T} \tau_{\mathrm{NC}}
\end{aligned}
$$

The square of the torque is a positive magnitude proportional to the electrical power consumption of the motor. The total overall torque is obtained summing $\mathrm{OT}_{\mathrm{CP}}$ for all the configurations in the workspace

$$
\mathrm{TOT}_{C}=\sum_{P \in \mathrm{WS}} \mathrm{OT}_{\mathrm{CP}}, \quad \mathrm{TOT}_{\mathrm{NC}}=\sum_{P \in \mathrm{WS}} \mathrm{OT}_{\mathrm{NCP}}
$$

To optimize the reduction of the overall torque for a given applied force, we first discretize the workspace of the manipulator. We set as parameters the stiffness constants of the springs and their resting positions $\left(k_{j}, \delta_{j}\right)$, for $j=1, \ldots, m$. At each configuration, we solve the system in Eq. (15). As it is a linear system, the system can be solved analytically, and then used to compute $\mathrm{OT}_{\mathrm{CP}}$. In each configuration, $\mathrm{OT}_{\mathrm{CP}}$ is a polynomial expression of the parameters $\left(k_{j}, \delta_{j}\right)$ that is always positive. The sum of all the polynomials $\mathrm{OT}_{\mathrm{CP}}\left(k_{j}, \delta_{j}\right)$ for all the configurations of the workspace gives $\operatorname{TOT}_{C}\left(k_{i}, \delta_{j}\right)$, which is also an always positive polynomial and can be minimized.

If several forces are considered, for example a discretization of a cone of forces, we can repeat the computation of the sum of all the $\mathrm{OT}_{C}\left(k_{j}, \delta_{j}\right)$ in each configuration of the workspace for each of the forces in the cone. The minimization of the resulting sum gives the spring parameters that better reduce the overall actuation torque for the forces inside the cone.

We used Wolfram Mathematica 9 for the simulations running under Windows 64bit, 16GB RAM. As a preliminary computation, we need to compute the workspace, which requires solving the inverse kinematics for each tested configuration, and thus, depending on the architecture it can be simpler or more expensive. Given the workspace, deducing the expression of $\mathrm{TOT}_{C}\left(k_{i}, \delta_{j}\right)$ takes about $0.054 \mathrm{~s}$ per configuration. Therefore, the computation time depends on the discretization of the workspace. Once the expression of $\operatorname{TOT}_{C}\left(k_{i}, \delta_{j}\right)$ is obtained, we use the Mathematica NMinimize() procedure to obtain the minimum of the functions for a given set of constraints.

It is important to realize that, by definition, the springs cannot reduce the overall torque for all the possible applied forces. But in general, a parallel manipulator will be operating in a specific region of the workspace and subjected to a subset of all possible forces specified for the required task and gravity. As an example, a flight simulator will have to primarily overcome a vertical force in the direction of gravity.
We measure the increase or decrease of exerted motor forces in a configuration by computing the percentage

$$
\frac{\mathrm{OT}_{\mathrm{CP}}-\mathrm{OT}_{\mathrm{NCP}}}{\mathrm{OT}_{\mathrm{NCP}}} 100 \%
$$

As a measure of the net improvement through all the workspace, we define the net percentage of increase/decrease as

$$
\frac{\mathrm{TOT}_{C}-\mathrm{TOT}_{\mathrm{NC}}}{\mathrm{TOT}_{\mathrm{NC}}} 100 \%
$$

The overall torque gives an idea of the electrical power consumption, but the limits of the workspace are usually limited by the maximum exerted motor force/torque. For this reason, we define an additional metric of motor force/torque as the maximum exerted in a configuration $P$ as

$$
\begin{aligned}
& \operatorname{MT}_{\mathrm{CP}}=\operatorname{Max}\left[\tau_{C}\right] \\
& \mathrm{MT}_{\mathrm{NCP}}=\operatorname{Max}\left[\tau_{\mathrm{NC}}\right]
\end{aligned}
$$

and the total maximum motor force/torque across the workspace as

$$
\mathrm{TMT}_{C}=\sum_{P \in \mathrm{WS}} \mathrm{MT}_{\mathrm{CP}}
$$

Then, similarly as before, the percentage of increase or decrease of maximum motor torque in a configuration is defined as

$$
\frac{\mathrm{MT}_{\mathrm{CP}}-\mathrm{MT}_{\mathrm{NCP}}}{\mathrm{MT}_{\mathrm{NCP}}} 100 \%
$$

and the net increase as

$$
\frac{\mathrm{TMT}_{C}-\mathrm{TMT}_{\mathrm{NC}}}{\mathrm{TMT}_{C}} 100 \%
$$

Note that the maximum torque is not a continuous function, and therefore, it cannot be minimized as done with the overall torque. Instead, we use the maximum motor torque as a measure to evaluate the results obtained minimizing the overall torque.

We use these performance measures in Secs. 4 and 5 to compute the optimal parameters for the springs of two examples of parallel manipulators.

\section{Example Application I: The Stewart-Gough Platform}

Consider the Stewart-Gough platform in Fig. 3-top. In Ref. [31] it was shown how this design is kinematically equivalent to the well-known octahedral design [32], having the same forward kinematic solution and the same singularities.

The base and platform attachments in their local reference are named $\boldsymbol{P}_{i}$ and $\boldsymbol{Q}_{i}$, respectively. The position and orientation of the platform are given by a position vector $\boldsymbol{p} \in \mathbb{R}^{3}$ and a rotation matrix $\boldsymbol{R} \in \mathrm{SO}(3)$. Then, the coordinates of the attachments with respect to the fixed reference frame located at the center of the base are $\boldsymbol{p}_{i}=\boldsymbol{P}_{i}$ and

$$
\boldsymbol{q}_{i}=\boldsymbol{p}+\boldsymbol{R} \boldsymbol{Q}_{i}
$$

The proposed design has the attachments in the base and in the platform aligned in a way that we can write

$$
\begin{aligned}
& \boldsymbol{p}_{2 i}=\lambda \boldsymbol{p}_{2 i-1}+(1-\lambda) \boldsymbol{p}_{2 i+1}, \quad \text { and } \\
& \boldsymbol{q}_{2 i+1}=\lambda \boldsymbol{q}_{2 i}+(1-\lambda) \boldsymbol{q}_{2 i+2}
\end{aligned}
$$




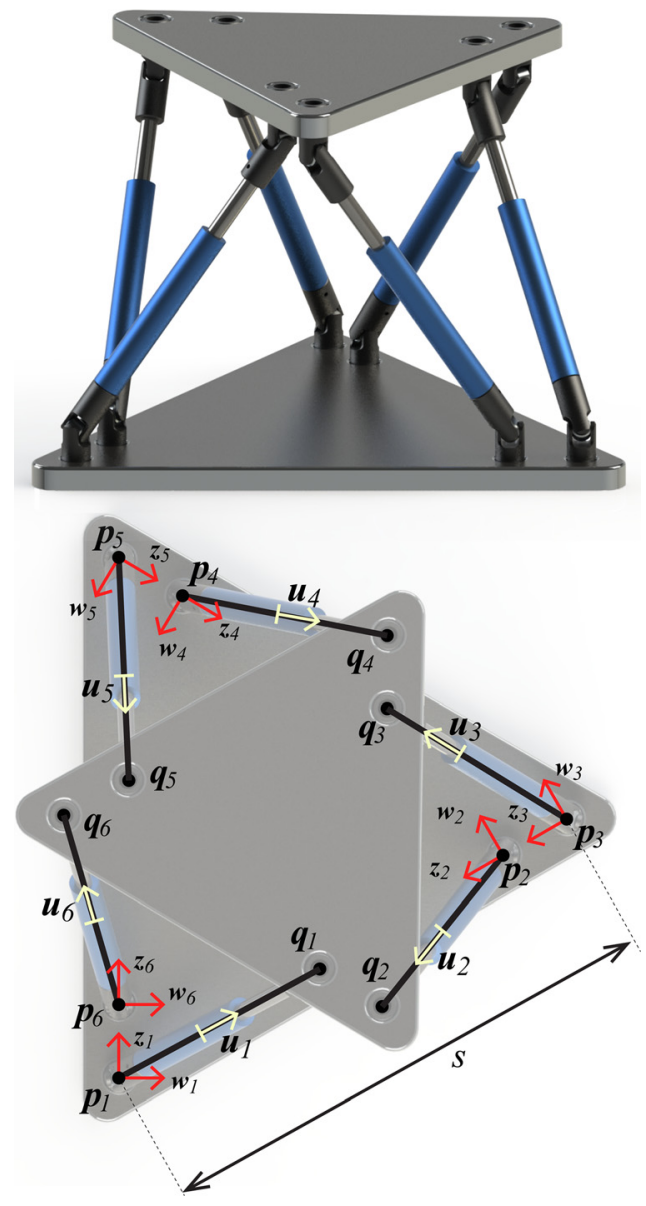

Fig. 3 Stewart-Gough platform equivalent to the octahedral design (top) and its corresponding notation (bottom). See Table 1 for coordinates of vectors $z_{i}$ and $w_{i}$.

Table 1 Joint axes coordinates for the Stewart-Gough platform

\begin{tabular}{lcc}
\hline \hline Leg $\boldsymbol{i}$ & First axis & Second axis \\
\hline $\boldsymbol{i}=1,6$ & $z_{i}=\left(\boldsymbol{p}_{5}-\boldsymbol{p}_{1}\right) / s$ & $\boldsymbol{w}_{\boldsymbol{i}}=z_{\boldsymbol{i}}^{\perp}$ \\
$\boldsymbol{i}=2,3$ & $z_{i}=\left(\boldsymbol{p}_{1}-\boldsymbol{p}_{3}\right) / s$ & $\boldsymbol{w}_{\boldsymbol{i}}=z_{i}^{\perp}$ \\
$\boldsymbol{i}=4,5$ & $z_{i}=\left(\boldsymbol{p}_{3}-\boldsymbol{p}_{5}\right) / s$ & $\boldsymbol{w}_{\boldsymbol{i}}=\boldsymbol{z}_{\boldsymbol{i}}^{\perp}$ \\
\hline \hline
\end{tabular}

for $i=1,2,3$ and indexes computed modulus 6 , where $\lambda$ represents the distance between the aligned attachments (see Fig. 3).

In each leg $i$, the first and second joints are the two DOFs that compose the universal joint, with axes $\boldsymbol{z}_{i}$ and $\boldsymbol{w}_{i}$, and angles $\theta_{i 1}$ and $\theta_{i 2}$, respectively, and the third joint corresponds to the prismatic joint following the direction of the unit vector $\boldsymbol{u}_{i}=\left(\boldsymbol{q}_{i}-\boldsymbol{p}_{i}\right) / l_{i}$ (Fig. 3-bottom). The forth, fifth, and sixth joints of each leg are composed of three intersecting rotational joints forming the platform spherical attachments (see Fig. 3 and Table 1 for detailed description of the axes).

4.1 Kinematic Analysis and Jacobian Matrix. The forward and inverse kinematics can be obtained solving the system

$$
\left(\boldsymbol{p}_{i}-\boldsymbol{q}_{i}\right)^{2}=l_{i}^{2}, \quad \text { for } \quad i=1, \ldots, 6
$$

where $l_{i}$ are the lengths of the legs.

It is well known that the Jacobian matrix of the Stewart-Gough platform is formed by the line screws defined by the legs. However, in this example, we consider compliance in parallel to the actuated prismatic joints and also in the passive universal joints at the base. As a result, we need to compute the Jacobian matrix of the corresponding redundant manipulator.

For each leg, we choose screws reciprocal to the 4th, 5th, and 6 th joints, and two additional joints. Then, for each leg $i$, the set of reciprocal screws are

$$
\begin{aligned}
& \$_{r i 1}=\left(\boldsymbol{u}_{i} \times \boldsymbol{w}_{i}, \boldsymbol{q}_{i} \times\left(\boldsymbol{u}_{i} \times \boldsymbol{w}_{i}\right)\right) \\
& \$_{r i 2}=\left(\boldsymbol{u}_{i} \times \boldsymbol{z}_{i}, \boldsymbol{q}_{i} \times\left(\boldsymbol{u}_{i} \times \boldsymbol{z}_{i}\right)\right) \\
& \$_{r i 3}=\left(\boldsymbol{u}_{i}, \boldsymbol{q}_{i} \times \boldsymbol{u}_{i}\right)
\end{aligned}
$$

We can build the 6 matrices $\boldsymbol{J}_{T i}$ following Eq. (6) and the above definition of reciprocal screws. The corresponding matrix in Eq. (7) gives

$$
\boldsymbol{J}_{\Theta \mathrm{i}}=\left(\begin{array}{ccc}
-q_{i z} & 0 & 0 \\
0 & q_{i z} & 0 \\
0 & 0 & 1
\end{array}\right)
$$

where $q_{i z}$ stands for the 3 th coordinate of the point $\boldsymbol{q}_{i}$. Following the definitions in Eqs. (9) and (10), $\boldsymbol{J}_{T}$ is a $18 \times 6$ matrix, and $\boldsymbol{J}_{\Theta}$ has dimensions $18 \times 18$. Therefore, the matrix $\boldsymbol{J}^{T}$ is a $6 \times 18$ matrix.

Only 6 motors are used, located at the prismatic joints, therefore, Eq. (15) has the form

$$
-\boldsymbol{W}=\boldsymbol{J}_{m}\left(\begin{array}{c}
f_{1} \\
\vdots \\
f_{6}
\end{array}\right)+\boldsymbol{J}^{T}\left(\begin{array}{c}
\vdots \\
k_{1}\left(\theta_{i 1}-\delta_{1}\right) \\
k_{2}\left(\theta_{i 2}-\delta_{2}\right) \\
k_{3}\left(l_{i}-l_{0}\right) \\
\vdots
\end{array}\right)
$$

where $\boldsymbol{J}_{m}$ is the usual $6 \times 6$ Jacobian where each column $i$ is the screw $\$_{r i 3}$ defined in Eq. (27), and the vector of forces and torques done by the springs is defined as in Eq. (1). where $k_{i}$ are the stiffness constants of the springs at the first, second, and third joints of each leg, respectively, $\delta_{i}, i=1,2$, the resting angle of the torsional springs located in parallel with the axes of the universal joint, and $l_{0}$ is the resting length of the linear spring located in parallel with the prismatic joint.

4.2 Results. All the simulations are computed for a manipulator with base side $1 \mathrm{~m}$ and platform side $0.75 \mathrm{~m}$. The offsets of the base and platform attachments correspond to $\lambda=0.1 \mathrm{~m}$ in Eq. (25).

We discretize the workspace in position and orientations as follows. Consider the vector $\Lambda$ orthogonal to the platform plane. We define the rotations of the platform as the cone of possible directions of the vector $\Lambda$ that spans two dimensions of possible rotations $\left(R_{x}\right.$ and $\left.R_{y}\right)$, where the maximum angle between two possible $\Lambda$ vectors represents the opening angle of the cone [33]. The additional rotation around $\Lambda$ represents the 3 th dimension of rotations $\left(R_{z}\right)$ (Fig. 4). We consider a discretization of the 6-dim workspace of 13, 247 configurations that include a range of directions of $\Lambda$ inside of a cone with opening angle of $5 \pi / 6 \mathrm{rad}$ and $R_{z}$ from $-\pi / 3$ to $\pi / 3$. The prismatic joint limits are $l_{i} \in[0.5,1]$, and the universal joint limits are $\theta_{i j} \in(-\pi / 3, \pi / 3)$.

To optimize the spring parameters, we apply the method introduced in Sec. 3.2, with optimization constraints

$$
\begin{gathered}
k_{1}>0, \quad k_{2}>0, \quad k_{3}>0, \\
-\frac{\pi}{2}<\delta_{1}<\frac{\pi}{2},
\end{gathered}
$$



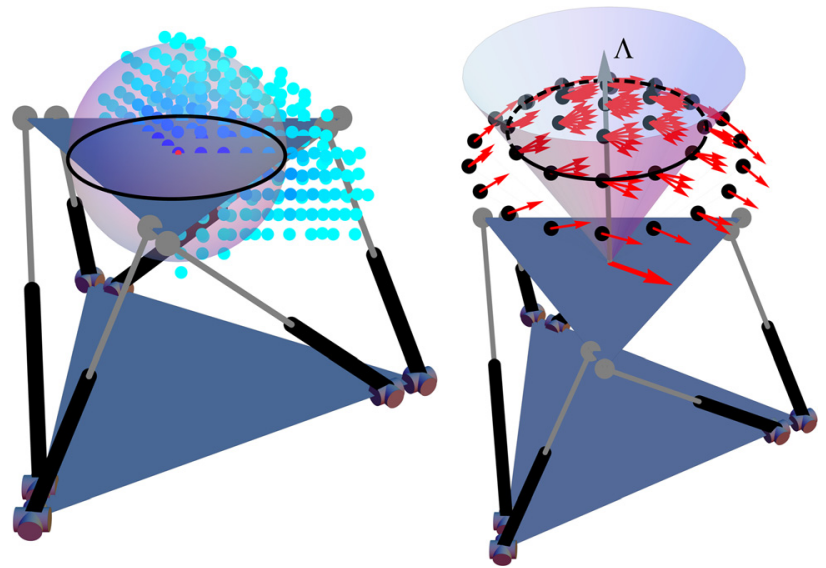

Fig. 4 Workspace discretization. (a) Position workspace. The points represent a third of the workspace, with darker color when more orientations are achievable. Points inside the sphere are considered part of the central workspace. (b) Orientation workspace for the shown position. Each dot represents an orientation of the vector Lambda, that is perpendicular to the platform. The red arrows show an additional rotation around Lambda. All orientations of Lambda inside the cone showed are considered part of the central workspace.

$$
\begin{gathered}
-\frac{\pi}{2}<\delta_{2}<\frac{\pi}{2}, \\
0.5<l_{0}<1.5
\end{gathered}
$$

For the optimization, we only consider configurations where the platform position vectors are inside the sphere shown in Fig. 4-left and the orientation inside the cone in Fig. 4-right. We denote the interior of the sphere and the orientations inside the cone as the central workspace, and focus efforts on the reduction of motor force in these workspace regions. With a single applied force of $\boldsymbol{W}=(0,0,-10,0,0,0)$, the obtained optimum is

$$
\begin{aligned}
& k_{1}=0.52, \quad k_{2}=1.31, \quad k_{3}=1.68, \\
& \delta_{1}=-0.92, \quad \delta_{2}=-0.03, \quad 1_{0}=1.5
\end{aligned}
$$

Considering a collection of applied forces on the border of a cone with opening angle of $2 \pi / 3 \mathrm{rad}$ (shown as black arrows in Fig. 6), all of magnitude 10, the optimization gives

$$
\begin{aligned}
& k_{1}=0.290, \quad k_{2}=0.725, \quad k_{3}=0.93, \\
& \delta_{1}=-0.916, \quad \delta_{2}=-0.03, \quad 1_{0}=1.5
\end{aligned}
$$

In Fig. 5(a), we show the computation of the overall reduction of the actuation torque using the compliant parameters in Eq. (31). The net percentage of increase of the overall torque, computed using Eq. (19) for an applied force $W=(0,0,-10,0,0,0)$ is $-84.08 \%$. Figure $5(a)$ top shows the histogram of the percentages of increase in all configurations of the WS. At the bottom, each position dot is plotted with a color according to the mean value of percentage of increase for all the achievable orientations from the position. Note that all the configurations of the workspace experience a reduction for this applied force.

If we assume that the motors can exert half of the force applied at the platform (that is, $5 \mathrm{~N}$ for an expected load of $10 \mathrm{~N}$ ), the static workspace is defined by those configurations that do not reach that limit. Without compliance, the static workspace is $71.9 \%$ of the kinematic workspace. Using compliance, it is $91.7 \%$ of the kinematic workspace. Figure $5(b)$ shows the distribution of the maximum torque over the workspace with and without compliance, and a representation of the position static workspace.
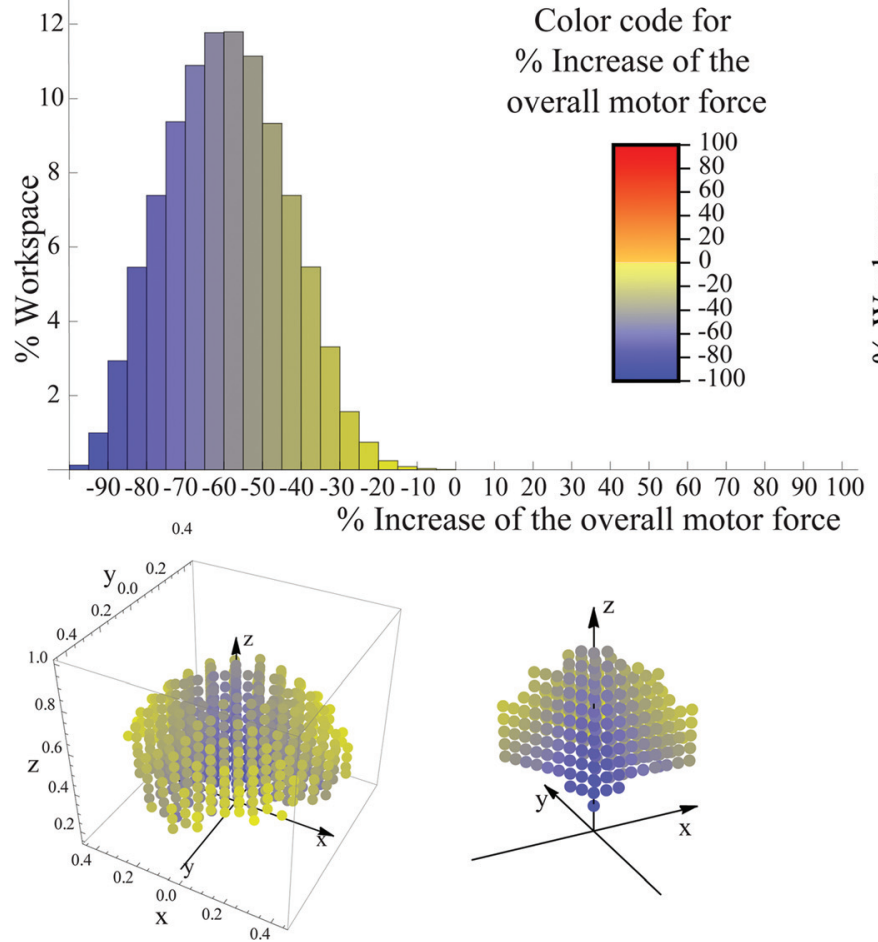

(a)

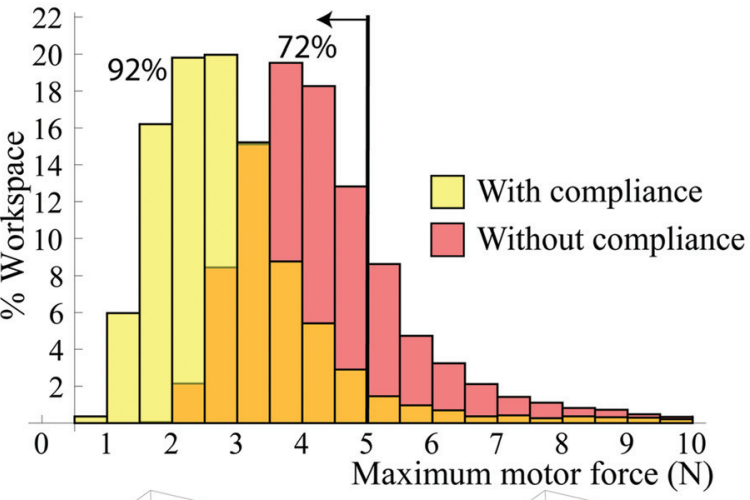

Without compliant joints

With compliant joints

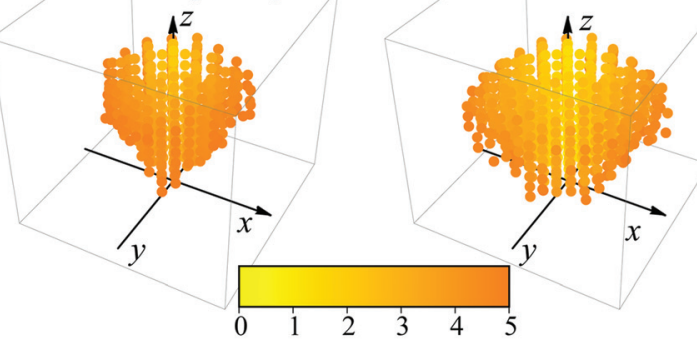

(b)

Fig. 5 (a) Top: Histogram of the percentage of increase/decrease of the motor force for each configuration in the workspace. Bottom representation of the position workspace, and a third of it. The colors represent the mean percentage of reduction for all the orientations achievable from each dot position. (b) Top: Comparison between histograms of the maximum torque values over the configurations of the workspace with and without compliant joints. Bottom: representation of the static workspace. Each dot color represents the median torque for all the possible orientations in the corresponding position of the dot. All points where the maximum force is bigger than $\mathbf{5}$ are discarded. 

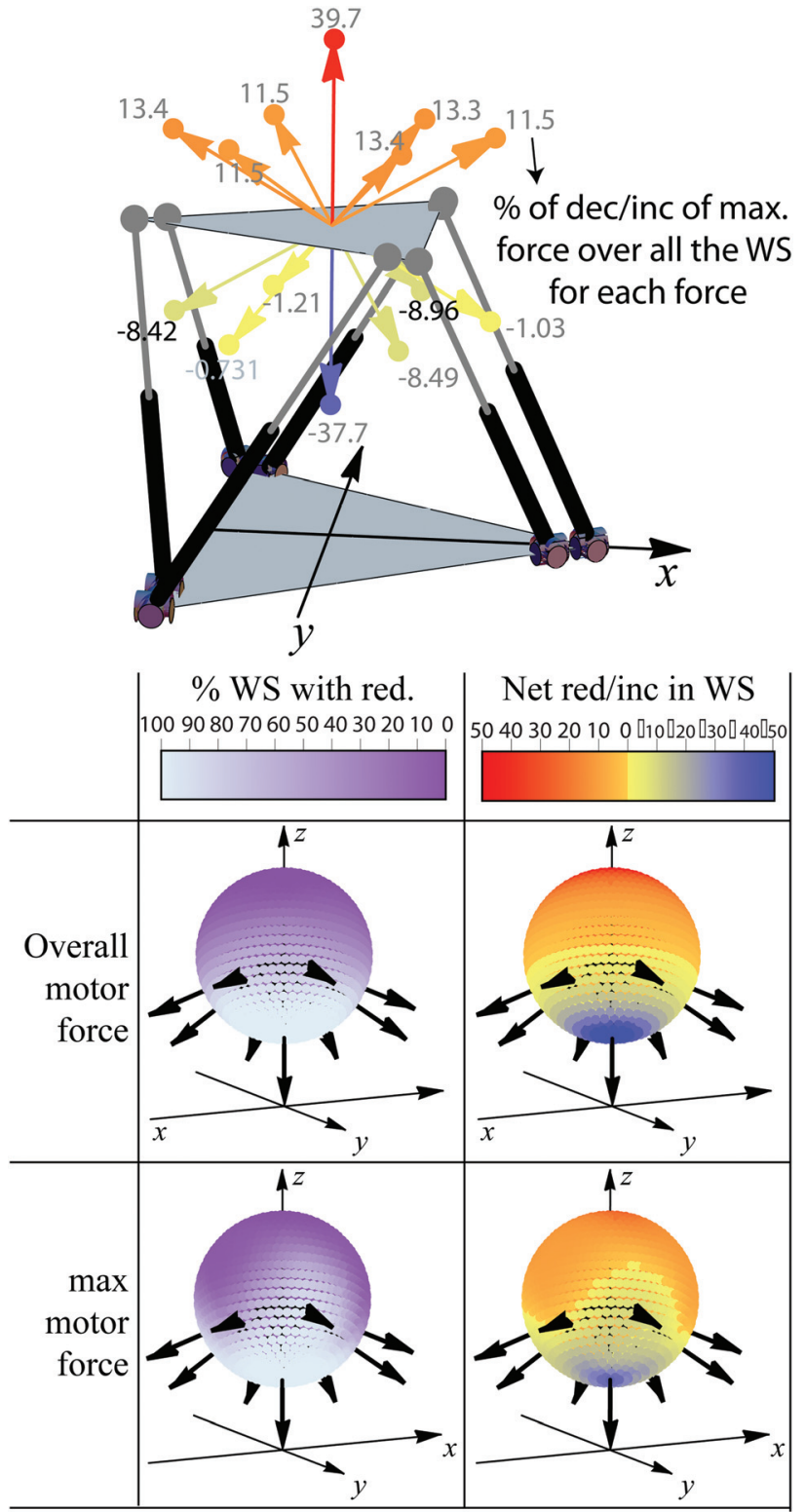

Fig. 6 Top: Each applied force is associated with a dot in a sphere. Bottom: The colors represent the percentage of the workspace with reduction (first column) or the net increase (second column) of the overall torque in the first row, or the maximum motor torque in the second row.

Figures 6 and 7 show how the increase of actuation force changes when different external forces are applied. Net increases and decreases are computed with respect the central workspace, that is, the amount of workspace inside the sphere and cone of orientations shown in Fig. 4.

In Fig. 6-top, we show a manipulator using the spring parameters in Eq. (31). Each arrow represents a direction of applied external force with magnitude $10 \mathrm{~N}$. The number next to the arrow represents the net percentage of increase of the maximum exerted torque over the central workspace, computed using Eq. (22). The colors follow the color code of the second bar (reds/oranges for positive increases, and yellows/blues for negative increases, i.e., decreases). In the spheres in Fig. 7-bottom, we only plot the tip dot of the arrows, and thus, each dot corresponds to an applied force with direction going from the center to the sphere to the dot, with magnitude $10 \mathrm{~N}$. In the first column of Fig. 7-bottom, each color represents the percentage of workspace where there is a reduction of the overall motor force (first row) or the maximum exerted motor force (second row). In the second column the colors represent the total percentage of increase of the overall torque using Eq. (19) (first row) and the percentage of increase of the maximum exerted motor force using Eq. (23) (second row). With this sphere representation, we can see what range of forces results in a net reduction of motor force.

In Fig. 7, the top plot shows percentages of workspace where there is a decrease of the overall torque when the magnitude of the force changes. The middle plot shows the mean decrease in this region and the bottom plot the overall net decrease using Eq. (19) in the central workspace. In all the plots, the $x$-axis represents the magnitude of the applied force, which direction is identified depending on the color, following the chart in the figure. Solid lines correspond to a manipulator optimized for a range of forces (parameters in Eq. (31)) while dashed lines are results for a manipulator optimized for a single force (parameters in Eq. (30)). The first manipulator gets bigger portions of workspace with reduction when the resultant applied force is not vertical.

\section{Example Application II: The 3-URS Platform}

Consider the manipulator in Fig. 8-top. It is a 6 DOF manipulator with 3 equal legs consisting of 2 links and 3 rotational joints each. For each leg $i, z_{i 1}=(0,0,1)^{T}$ is the axis of rotation of the first joint, with rotation angle $\theta_{i 1}$. The axis of rotation of the second joint is $z_{i 2}=\left(\sin \left(\theta_{i 1}\right),-\cos \left(\theta_{i 1}\right), 0\right)^{T}$ with a rotation angle $\theta_{i 2}$. Finally, the third axis is parallel to the previous one, with angle of rotation $\theta_{i 3}$.

5.1 Kinematic Analysis and Jacobian Matrix. As in the previous example, the coordinates of the attachments, with respect to the fixed reference frame located at the center of the base, are $\boldsymbol{p}_{i}=\boldsymbol{P}_{i}$ and

$$
\boldsymbol{q}_{i}=\boldsymbol{p}+\boldsymbol{R} \boldsymbol{Q}_{i}, \quad i=1,2,3
$$

Alternatively, the coordinates of the platform attachments can also be parameterized with respect to the angles of rotation of the joint angles as

$$
\boldsymbol{q}_{i}=\boldsymbol{p}_{i}+s_{i}(0,0,1)^{T}+r_{i}\left(\cos \left(\theta_{i 1}\right), \sin \left(\theta_{i 1}\right), 0\right)^{T}
$$

where

$$
\begin{aligned}
& s_{i}=l_{i} \sin \left(\theta_{i 2}\right)+d_{i} \sin \left(\theta_{i 2}+\theta_{i 3}\right), \\
& r_{i}=l_{i} \cos \left(\theta_{i 2}\right)+d_{i} \cos \left(\theta_{i 2}+\theta_{i 3}\right)
\end{aligned}
$$

and $l_{i}$ and $d_{i}$ are the lengths of the links of the $i$ th leg. We can obtain similar parameterization of the center points of the joints $\theta_{i 3}[24]$.

The loop equations are the 9 equations obtained by equating the platform attachment coordinates computed with respect to the position and orientation of the platform $\boldsymbol{q}_{i}^{x}$ (Eq. (32)) with the same coordinates computed using the joint angles $\boldsymbol{q}_{i}^{a}$ (Eq. (33)).

The manipulator has 3 legs with 2 links each, and a total of 6 joints per leg ( 2 in the base universal joint, 1 in the rotational joint and 3 in the platform attachment spherical joint). The mobility of the platform is 6 (full mobility) and therefore, only 6 motors are needed to fully actuate the platform in all its degrees of freedom. We consider all joints compliant except the platform attachments, with only two motors per leg, located at the two base joints.

The Jacobian matrix $\boldsymbol{J}^{T}$ can be computed using screw theory following the steps proposed Sec. 2.2. In this case, the Jacobian matrices are

$$
\boldsymbol{J}_{\Theta i}^{-1}=\left(\begin{array}{ccc}
\frac{-1}{l_{i} \cos \left(\theta_{i 2}\right)+d_{i} \sin \left(\theta_{i 2}+\theta_{i 3}\right)} & 0 & 0 \\
0 & \frac{1}{l_{i} d_{i} \sin \left(\theta_{i 3}\right)} & 0 \\
0 & 0 & \frac{-1}{l_{i} d_{i} \sin \left(\theta_{i 3}\right)}
\end{array}\right)
$$



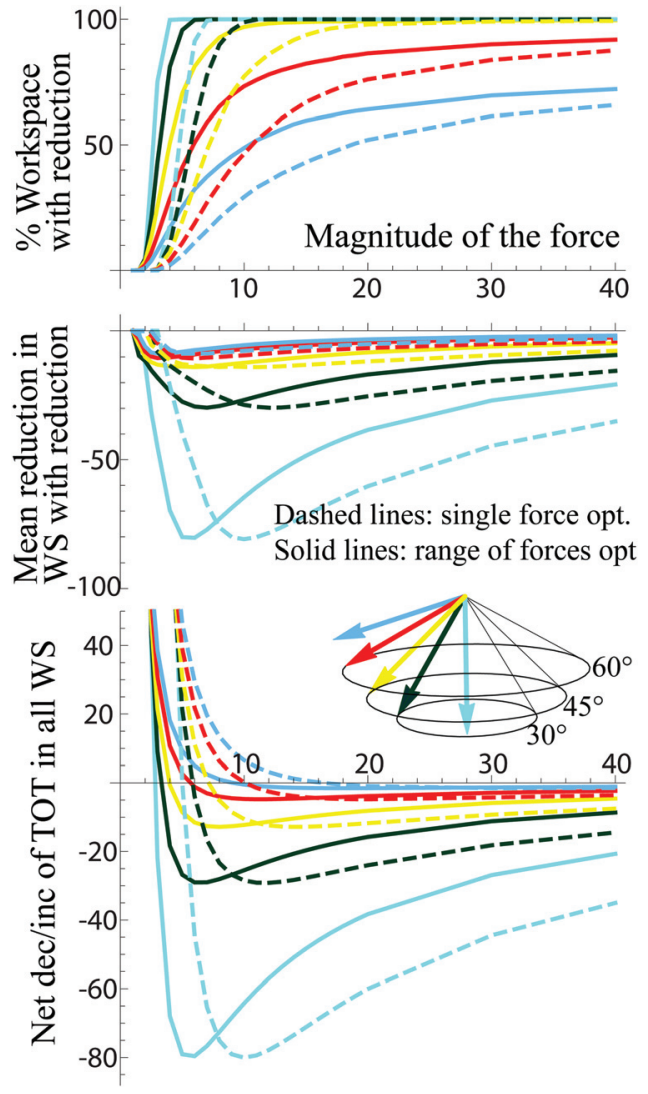

Fig. 7 Percentages of reduction of the motor torque when changing the magnitude of the applied force. Each color corresponds to a different direction of applied force. Dashed (solid) lines are results of a manipulator using the compliant parameters in Eqs. (30) and (31).

and $J_{T}=\left(\xi_{i j}\right)^{T}$ is a matrix where each row $\xi_{i j}$ is defined as

$$
\begin{aligned}
& \boldsymbol{\xi}_{i 1}=\left(z_{i 2}, \quad \boldsymbol{q}_{i} \times z_{i 2}\right)^{\boldsymbol{T}} \\
& \boldsymbol{\xi}_{i 2}=\left(\boldsymbol{q}_{i}-\boldsymbol{t}_{i}, \quad \boldsymbol{q}_{i} \times\left(\boldsymbol{q}_{i}-\boldsymbol{t}_{i}\right)\right)^{\boldsymbol{T}} \\
& \boldsymbol{\xi}_{i 3}=\left(\boldsymbol{q}_{i}-\boldsymbol{p}_{i}, \quad \boldsymbol{q}_{i} \times\left(\boldsymbol{q}_{i}-\boldsymbol{p}_{i}\right)\right)^{\boldsymbol{T}}
\end{aligned}
$$

for $i=1,2,3$, where the vector $z_{i 2}=\left(\sin \left(\theta_{i 1}\right),-\cos \left(\theta_{i 1}\right), 0\right)^{T}$ has been introduced before as the second joint axis of rotation and $\boldsymbol{t}_{i}$ are the center points of the third joints at each leg $i$.

Note that the Jacobian matrix $\boldsymbol{J}=\boldsymbol{J}_{\Theta}^{-1} \boldsymbol{J}_{T}$ is a $9 \times 6$ matrix and the static equilibrium equation in Eq. (14) has a vector of compliant torques

$$
\tau_{c}=\left(\begin{array}{c} 
\\
\vdots \\
k_{1}\left(\theta_{i 1}-\delta_{i 1}\right) \\
k_{2}\left(\theta_{i 2}-\delta_{2}\right) \\
k_{3}\left(\theta_{i 3}-\delta_{3}\right) \\
\vdots
\end{array}\right)
$$

for $i=1,2,3$, where $k_{j}$ are the 3 spring constants and $\delta_{i 1}, \delta_{2}$, and $\delta_{3}$ the five parameters for the spring free lengths. We omit the sub-index $i$ for the spring parameters that are equal for each leg.

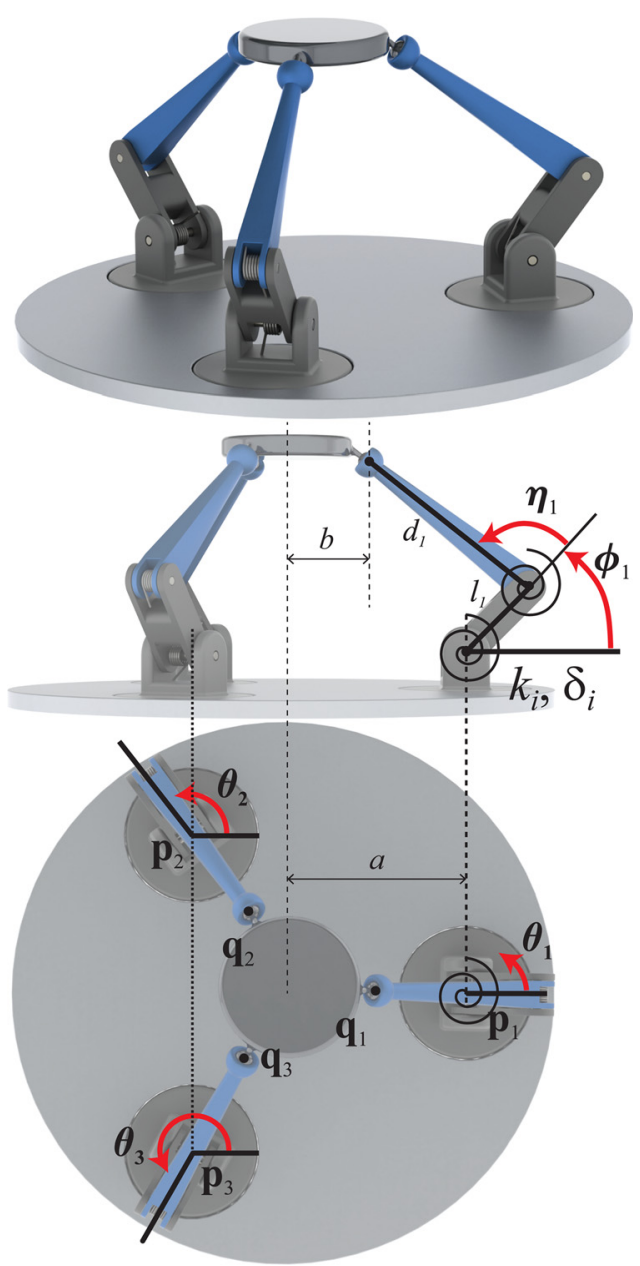

Fig. 8 Notation for the 3-URS manipulator

The vector of motor torques contain only 6 nonzero elements, because $\tau_{i 3}=0$ for $i=1,2,3$. Then, the static equilibrium system becomes square as in Eq. (15).

5.2 Results. For the simulations, we considered a manipulator with dimensions $a=0.7, b=0.2, l_{i}=1 / 3$, and $d_{i}=2 / 3$, for $i=1,2,3$. Using the same discretization of the workspace as in the previous example, we solve the inverse kinematics in each configuration to get the corresponding angle joints. The spherical attachments are considered with a range of motion forming a cone with opening angle of $140 \mathrm{deg}$.

For the results in all the tables, we discard the configurations too close to a singularity, that is, where the determinant of the Jacobian matrix is smaller than $10^{-5}$

Following similar steps to the previous example, we apply the optimization of the range of forces shown in black in Fig. 12 for forces of magnitude $10 \mathrm{~N}$. The results give

$$
\begin{aligned}
& k_{1}=0.94, \quad k_{2}=0.45, \quad k_{3}=0.54 \\
& \delta_{11}=0, \quad \delta_{12}=\frac{2 \pi}{3}, \quad \delta_{13}=\frac{4 \pi}{3} \\
& \delta_{2}=0, \quad \delta_{3}=0
\end{aligned}
$$

If we instead perform the same optimization for forces of magnitude $2 \mathrm{~N}$ (in the same directions), then the optimal parameters obtained are the same as before, except the spring constants are divided by 5 (proportional to the applied forces). 

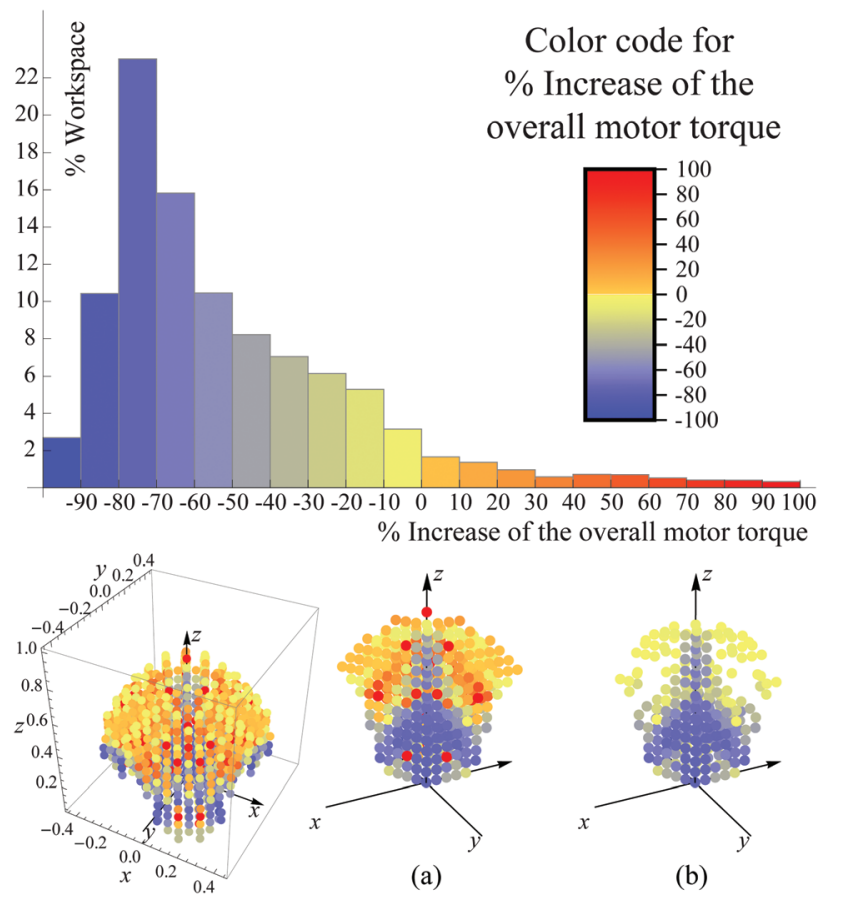

Fig. 9 Top: Histogram of the percentage of increase of the overall motor torque in each configuration of the workspace. Bottom: Each dot in the position workspace is colored depending on the mean reduction of the overall motor torque for all the orientations achievable from the dot position. From the left figure, (a) shows only a third to the figure, and $(b)$ only the third and only configurations with reduction.

Optimizing for a single force $(0,0,-10,0,0,0)$ gives

$$
\begin{aligned}
& k_{1}=1.69, \quad k_{2}=0.80, \quad k_{3}=0.98 \\
& \delta_{11}=0, \quad \delta_{12}=\frac{2 \pi}{3}, \quad \delta_{13}=\frac{4 \pi}{3} \\
& \delta_{2}=0, \quad \delta_{3}=0
\end{aligned}
$$

Figure 9 shows the distribution of configurations for which there is reduction of overall torque when a force $F=(0,0$, $-10,0,0,0)$ is applied, using Eq. (18). The color of each dot in the workspace represents the mean value of Eq. (18) for the configurations corresponding to all the possible orientations from the dot position. As a global measurement, using the spring parameters in Eq. (36), 88.4\% of the configurations in the workspace show a reduction of the overall torque, with a total reduction of $-66.32 \%$ in that region. The net reduction over all the workspace, computed as in Eq. (19), is $-58.25 \%$.

Figure 10 shows the modification of the static workspace. In this case, we set the limit of the motor torques to $10 \mathrm{Nm}$. When applying the vertical force $(0,0,-10,0,0,0)$, the manipulator without springs can reach only $26.77 \%$ of the kinematic workspace. With the springs, the reachable workspace increases to $47.67 \%$ of the kinematic workspace. Figure 10 shows a representation of the position static workspace and the histogram of the maximum motor torque in all the configurations of the workspace for the manipulator without springs (dark color/red) and the manipulator using the springs with parameters in Eq. (36) (light color/yellow).

Figures 11 and 12 show results where different applied forces are considered, showing reductions/increases of the motor torque for the central regions of the WS similarly as in the previous example. Figure 11 shows how results change when the magnitude of the applied force changes. Dashed lines show results for the optimum obtained with a range of applied forces of magnitude $2 \mathrm{~N}$, and solid lines optimums obtained with $10 \mathrm{~N}$ range of applied

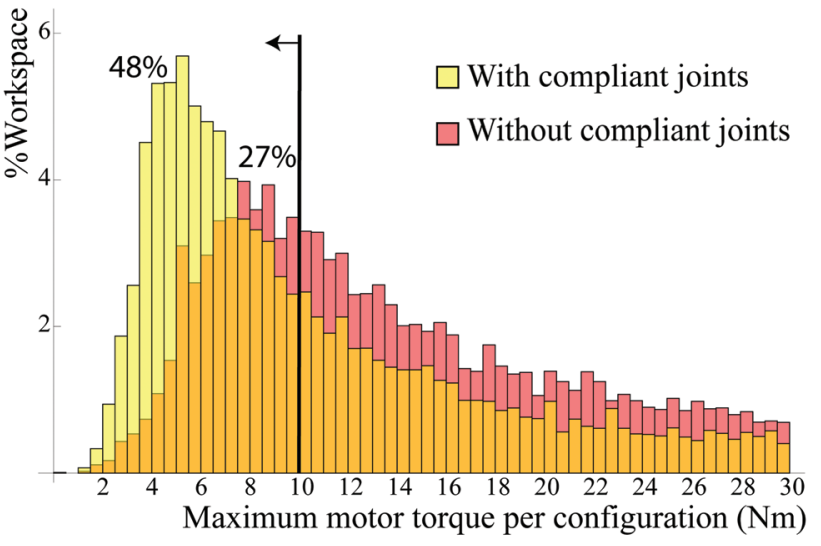

Without compliance

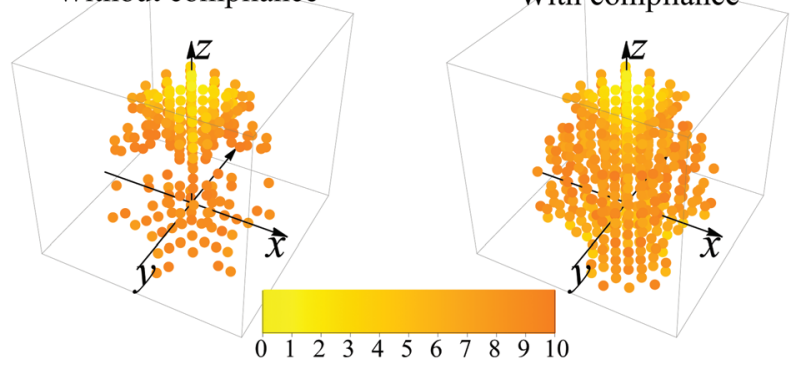

Fig. 10 Top: Comparison between histograms of the maximum torque values over the configurations of the workspace with and without compliant joints. Bottom: A representation of the static workspace. Each dot color represents the median torque for all the possible orientations in the corresponding position of the dot. All points where the maximum torque is bigger than $10 \mathrm{Nm}$ are discarded.

forces. Different colors correspond to different directions of the applied forces shown in the diagram.

Finally, Fig. 12 shows the reduction over the central workspace for all the possible applied force directions, for a manipulator using the parameters in Eq. (36). The net increases in all the WS are computed using Eq. (19) for the overall motor torque and Eq. (23) for the maximum motor torque.

\section{Discussion}

The results for the two parallel manipulators analyzed show that for a desired range of force directions applied on the platform, springs can help to significantly reduce the motor torque.

Figures 6 and 12 show that springs cannot reduce the motor torque for all possible applied forces, because if there is reduction of actuation force under one force direction, there will be increase in the opposite one. However, we have provided design tools to optimize the manipulator for the desired range of external forces, and the desired regions of the workspace where motor force reduction occurs. This allows users to specify the desired workspace regions and directions of forces for which the most significant motor torque reduction is needed.

If a manipulator has to perform tasks with a preferred direction of external applied forces, as for example a flight simulator, the static workspace, which defines the real borders of the reachable workspace, can be greatly increased by an appropriate selection of the springs. For a more versatile and general-use manipulator, springs in parallel with the motors may not be a good design solution.

The curves in Figs. 7 and 11 indicate that the performance under lower forces can significantly increase the motor torques instead of reducing it, but a significant reduction will occur for larger forces. Therefore, we can conclude that the springs have to be designed to compensate the minimum external force the manipulator has to resist, for instance, the weight of the platform. 


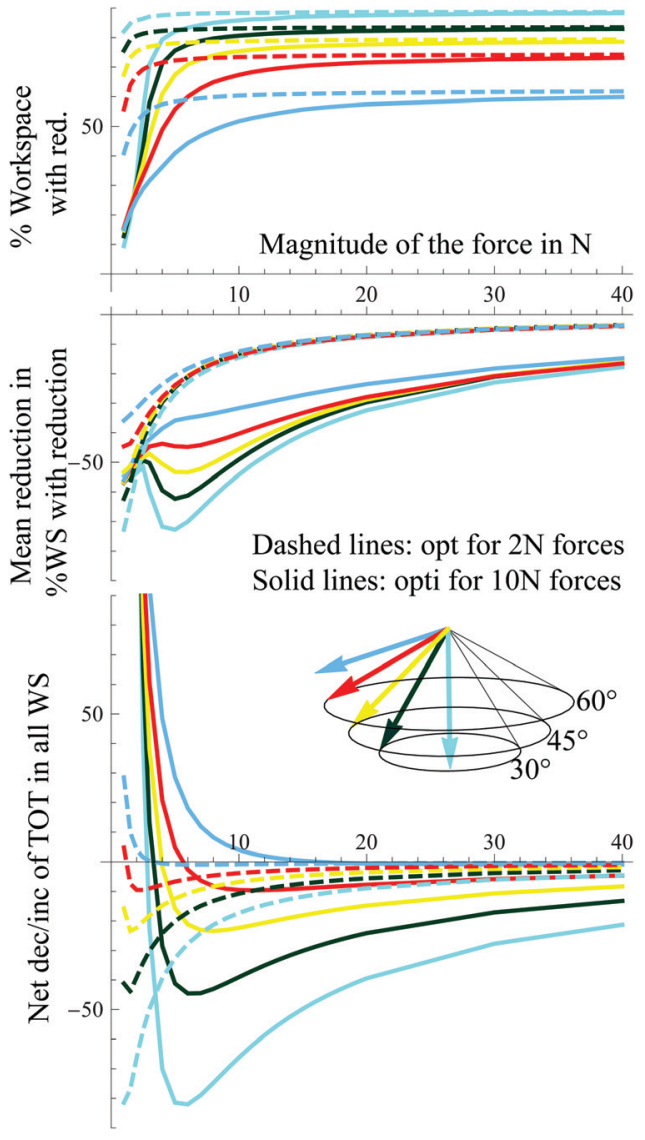

Fig. 11 Comparison of manipulators using the compliant parameters optimized for a range of forces of magnitude $10 \mathrm{~N}$ (Eq. (36)) and for a range of forces of magnitude $2 \mathrm{~N}$.

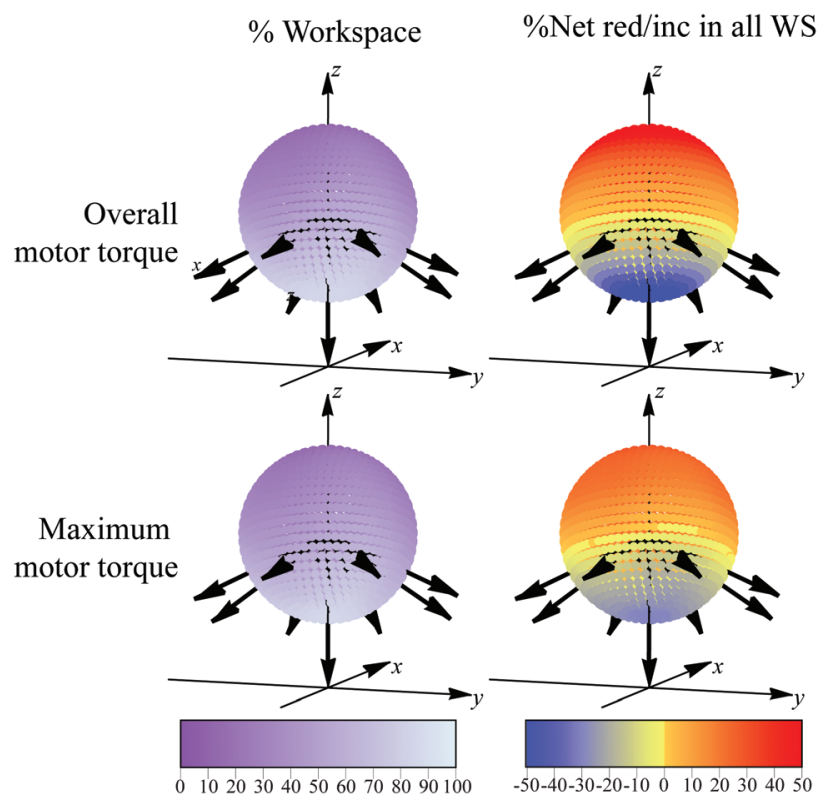

Fig. 12 Similarly to Fig. 6, each dot represents a direction of the external applied force, and its color the percentage of workspace with reduction (first column) and the net reduction (second column). Reductions of the overall torque are shown in the first row, while reductions of the maximum torque in each configuration are shown in the second row.
Other approaches to reduce actuation torque on parallel manipulators like static balance or gravity compensation methods require a complex design and an ad-hoc location of the compliant elements. However, we have shown that by simply locating springs in parallel with the motors, large reductions of actuation torque can be also obtained.

This simple solution to reduce actuation torque has been applied in industry for simple cases, as for instance, using springs in parallel with the prismatic motors of industrial electric jacks. Here, we provide a method to implement similar reductions for more complex architectures including active and passive (rotational and prismatic) joints.

\section{Conclusions}

We have presented a mathematical framework that clearly distinguishes between active and passive compliant joints. The framework can be used to model compliant parallel manipulators by changing the definition of the compliant actuated joint torque, with the spring serially connected to the motor as in Ref. [6]. In this case, the proposed formulation show simpler static equilibrium equations than previous approaches, as it only requires the computation of one Jacobian matrix instead of the Jacobian between passive and active joints.

The quantification of the variation of the torques due to the presence of passive compliance has been expressed in an analytical equation that allows us to study such variation through all the workspace of the manipulator.

The framework has been successfully applied to two examples of manipulators that show a significant reduction of the actuator forces for a significant range of forces and portions of the workspace.

In a future work, we want to study the influence of compliance when it acts in parallel with a pulling cable in underactuated hands manipulating objects [3]. In one torque direction, the compliance acts in parallel and thus, it behaves as in the proposed model, but in the other direction the cable becomes loose and the compliant joints become passively compliant.

The presented analysis, together with the existing compliant parallel robots analysis, constitutes a further step toward a full understanding of the role of compliant joints in parallel manipulators.

\section{Acknowledgment}

This work was supported in part by National Science Foundation grant IIS-0952856. A preliminary version of this paper is in the proceedings of the 2012 IEEE International Conference on Intelligent Robots and Systems (IROS) [24].

\section{References}

[1] Pratt, G. A., and Williamson, M. M., 1995, "Series Elastic Actuators," IEEE/ RSJ International Conference on Intelligent Robots and Systems, pp. 399-406.

[2] Dollar, A. M., and Howe, R. D., 2010, "The Highly Adaptive SDM Hand Design and Performance Evaluation," Int. J. Rob. Res., 29, pp. 585-597.

[3] Balasubramanian, R., Belter, J. T., and Dollar, A. M., 2012, "Disturbance Response of Two-Link Underactuated Serial-Link Chains," ASME J. Mech. Robot., 4(2), p. 021013.

[4] Pashkevich, A., Klimchik, A., and Chablat, D., 2011, "Enhanced Stiffness Modeling of Manipulators With Passive Joints," Mech. Mach. Theory, 46, pp 662-679.

[5] Pashkevich, A., Klimchik, A., and Chablat, D., 2010, "Stiffness Analysis of Parallel Manipulators With Preloaded Passive Joints," Advances in Robot Kinematics: Motion in Man and Machine, Springer Netherlands, The Netherlands, pp. $465-474$.

[6] Quennouelle, C., and Gosselin, C. M., 2009, "A Quasi-Static Model for Planar Compliant Parallel Mechanisms," ASME J. Mech. Rob., 1(2), p. 021012

[7] Carbone, G., 2011, "Stiffness Analysis and Experimental Validation of Robotic Systems," Front. Mech. Eng., 6, pp. 182-196.

[8] Su, H.-J., and McCarthy, J. M., 2006, "A Polynomial Homotopy Formulation of the Inverse Static Analysis of Planar Compliant Mechanisms," ASME J. Mech. Des., 128(4), pp. 776-786.

[9] Gosselin, C. M., 2008, "Gravity Compensation, Static Balancing, and Dynamic Balancing of Parallel Mechanisms," Smart Devices and Machines for Advanced Manufacturing, pp. 27-48. 
[10] Jean, M., and Gosselin, C., 1996, "Static Balancing of Planar Parallel Manipulators," IEEE International Conference on Robotics and Automation, pp. $3732-3737$.

[11] Wang, J., and Gosselin, C., 1999, "Static Balancing of Spatial Three-Degreeof-Freedom Parallel Mechanisms," Mech. Mach. Theory, 34, pp. 437-452.

[12] Merlet, J. P., 2006, Parallel Robots, 2nd ed., Springer, New York.

[13] Hubert, J., and Merlet, J. P., 2008, "Singularity Analysis Through Static Analysis," Adv. Rob. Kinematics, Springer Netherlands, pp. 13-20.

[14] Hubert, J., and Merlet, J. P., 2009, "Static of Parallel Manipulators and Closeness to Singularity," ASME J. Mech. Rob., 1(1), p. 011011.

[15] Quennouelle, C., and Gosselin, C., 2011, "Kinematostatic Modeling of Compliant Parallel Mechanisms. Application to a 3-PRRR Mechanism, the Tripteron," Meccanica, 46, pp. 155-169.

[16] Carbone, G., and Ceccarelli, M., 2004, "A Stiffness Analysis for a Hybrid Parallel-Serial Manipulator,” Robotica, 22, pp. 567-576.

[17] Gosselin, C., 1990, "Stiffness Mapping for Parallel Manipulators," IEEE Trans. Robot. Autom., 6, pp. 377-382.

[18] Pashkevich, A., Chablat, D., and Wenger, P., 2009, "Stiffness Analysis of Overconstrained Parallel Manipulators," Mech. Mach. Theory, 44, pp. 966-982.

[19] Quennouelle, C., and Gosselin, C., 2008, "Stiffness Matrix of Compliant Parallel Mechanisms," International Design Engineering Technical Conferences \& Computers and Information in Engineering Conference, Brooklyn, New York.

[20] Schmit, N., and Okada, M., 2012, "Simultaneous Optimization of Robot Trajectory and Nonlinear Springs to Minimize Actuator Torque," IEEE International Conference on Robotics and Automation, RiverCentre, Saint Paul, MN, pp. $2806-2811$.

[21] Schauß, T., Scheint, M., Sobotka, M., Seiberl, W., and Buss, M., 2009, "Effects of Compliant Ankles on Bipedal Locomotion," IEEE International Conference on Robotics and Automation, Kobe, Japan, pp. 2761-2766.
[22] Mombaur, K. D., Longman, R. W., Bock, H. G., and Schlöder, J. P., 2005, "Open-Loop Stable Running," Robotica, 23, pp. 21-33.

[23] Dasgupta, B., and Mruthyunjaya, T. S., 1998, "Force Redundancy in Parallel Manipulators: Theory and Practical Issues," Mech. Mach. Theory, 33, pp. 727-742.

[24] Borràs, J., and Dollar, A. M., 2012, "Static Analysis of Parallel Robots With Compliant Joints for In-Hand Manipulation," International Conference on Intelligent Robots and Systems, Portugal.

[25] Kanaan, D., Wenger, W., Caro, S., and Chablat, D., 2009, "Singularity Analysis of Lower Mobility Parallel Manipulators Using Grassmann-Cayley Algebra," IEEE Trans. Rob., 25, pp. 995-1004.

[26] Mohamed, M. G., and Gosselin, C. M., 2005, "Design and Analysis of Kinematically Redundant Parallel ManipulatorsWith Configurable Platforms,” IEEE Trans. Rob., 21, pp. 277-287.

[27] Tsai, L.-W., 1999, Robot Analysis. The Mechanics of Serial and Parallel Manipulators, John Wiley \& Sons, Inc., New York. 633-651.

[28] Mohamed, M. G., and Duffy, J., 1985, "A Direct Determination of the Instantaneous Kinematics of Fully Parallel Robot Manipulators," J. Mech. Transm. Autom. Des., 107, pp. 226-229.

[29] Dai, J. S., Huang, Z., and Lipkin, H., 2006, "Mobility of Overconstrained Parallel Mechanisms," ASME J. Mech. Des., 128, pp. 220-229.

[30] Dai, J. S., and Jones, J. R., 2001, "Interrelationship Between Screw Systems and Corresponding Reciprocal Systems and Applications," Mech. Mach. Theory, 36, pp. 633-651.

[31] Borràs, J., Thomas, F., and Torras, C., "New Geometric Approaches to the Analysis and Design of Stewart-Gough Platforms," IEEE/ASME Trans. Mechatronics, (in press).

[32] Downing, D., Samuel, A., and Hunt, K., 2002, "Identification of the Special Configurations of the Octahedral Manipulator Using the Pure Condition," Int. J. Rob. Res., 21, pp. 147-159.

[33] Cone, 2013. Available: http://mathworld.wolfram.com/cone.html 